\title{
Association Between Brain Venous Drainage, Cerebral Aneurysm Formation and Aneurysm Rupture
}

\author{
Enes DUMAN ${ }^{1}$, Ilker COVEN², Erkan YILDIRIM¹, Cem YILMAZ², H. Ulas PINAR ${ }^{3}$, Ozgur OZDEMIR \\ ${ }^{1}$ Baskent University, Medical Faculty, Konya Research Center, Department of Radiology, Konya, Turkey \\ ${ }^{2}$ Baskent University, Medical Faculty, Department of Neurosurgery, Ankara, Turkey \\ ${ }^{3}$ Baskent University, Medical Faculty, Konya Research Center, Department of Anesthesiology, Konya, Turkey \\ ${ }^{4}$ Baskent University, Medical Faculty, Konya Research Center, Department of Neurosurgery, Konya, Turkey
}

\section{ABSTRACT}

AIM: The brain venous drainage dominance is generally divided into three groups; right or left dominance and co-dominance. There is no study in the literature examining the link between brain venous drainage and aneurysm formation or rupture. Our aim was to evaluate the association between venous dominancy, aneurysm formation and rupture.

MATERIAL and METHODS: Eighty-six patients, who underwent cerebral digital subtraction angiography and who had cerebral aneurysms, were included in the study. The angiographic images, patient charts, and tomography images were scanned retrospectively. We recorded the aneurysm's location, size, dome to neck ratio (D/N); the patient's gender, age, whether there was a ruptured aneurysm, smoking history, and/or hypertension; dominance of venous drainage, aneurysm side, Fisher scores and the World Federation of Neurosurgical Societies (WFNS) Grading System for Subarachnoid Hemorrhage scores for patients who had a ruptured aneurysm. We assessed whether or not venous drainage was associated with rupture of the aneurysm and if venous dominance was a predisposing factor for aneurysm formation like location, size, and hypertension.

RESULTS: There was a statistically significant association between venous dominance and side of aneurysm; and also a statistically significant association between venous dominance and rupture. There was a positive correlation between hypertension and rupture. The most common aneurysm location was the anterior communicating artery, followed by the middle cerebral artery.

CONCLUSION: Brain venous drainage dominance may be a predisposing factor for aneurysm formation and it can be predictive for rupture.

KEYWORDS: Cerebral aneurysm, Brain venous drainage, Venous dominance, Rupture

\section{INTRODUCTION}

S ubarachnoid hemorrhage (SAH) is a relatively rare problem and is responsible for $3 \%$ of all stroke cases and $5 \%$ of stroke-related deaths (1). Spontaneous nontraumatic SAH is usually due to saccular aneurysm rupture. Other rare causes are arteriovenous malformations, arterial dissections, tumors, or other vascular anomalies (8).

Risk factors for $\mathrm{SAH}$ are hypertension, smoking, using oral contraceptives, pregnancy, excessive alcohol use, using co- caine, diurnal changes in blood pressure, family history of $\mathrm{SAH}$ and aneurysm, genetic disorders, older ages, lumbar puncture and cerebral angiography in patients with aneurysm (7).

Aneurysmal subarachnoid hemorrhage (A-SAH) has high morbidity and mortality rates, while SAH due to other nonaneurysm rupture-related etiologies has a better prognosis (5). The life-threatening and debilitating consequences of SAH may be prevented if the aneurysm can be occluded before rupture. For effective management of patients with 
un-ruptured aneurysms, prognostic criteria for the risk of rupture are needed, for which aneurysm size, location, and multiplicity are key prognostic factors (14). As a means of providing an inclusive risk prediction model, the PHASES (Population, Hypertension, Age, Size of the aneurysm, Earlier subarachnoid hemorrhage from another aneurysm, and Site of the aneurysm) score, based on a pooled analysis of six prospective natural history studies, was recently proposed (4).

The cerebral venous system can be divided into a superficial and a deep system. The superficial system consists of sagittal sinuses and cortical veins and these drain the superficial parts of both cerebral hemispheres. The deep system comprises the lateral sinus, straight sinus and sigmoid sinus, along with deeper draining cortical veins (13).

The aim of this study was to review our patient population with $\mathrm{SAH}$ due to ruptured cerebral aneurysm, and to determine whether venous drainage is associated with rupture of aneurysm, and whether venous drainage dominance is a predisposing factor like location, size, hypertension, and smoking. We aimed to evaluate whether there was a relationship between aneurysm formation and venous drainage of the brain.

\section{MATERIAL and METHODS}

This study was approved by our University Institutional Review Board and supported by our University Research Fund.

Eighty-six patients, who underwent cerebral digital subtraction angiography (DSA) between 2011-2015 and had cerebral aneurysm, were included in the study. The angiographic images, patient charts, and tomography images were scanned retrospectively. The aneurysm's location, size, dome to neck ratio (D/N); gender, ages, if they had ruptured aneurysm, smoking and hypertension; dominance of venous drainage, aneurysm sides, Fisher scores and World Federation of Neurosurgical Societies Grading System for Subarachnoid Hemorrhage (WFNS) scores were recorded.

Aneurysm sides were divided into two groups, whether they were demonstrated from right-side injection or left-side injection. If the aneurysm was on the anterior communicating artery, the side of aneurysm was accepted according to its dominant filling side; if it was filling dominantly from right carotid injection, we accepted that the aneurysm side was 'right'; if it was filling from the left carotid injection, we accepted that the aneurysm side was 'left'. If it was filling from both side injections equally, we excluded these patients from the study. Patients who had dural venous pathology were also excluded from the study.

Angiographically, venous drainage dominance of dural venous sinuses is defined as the drainage of the radio-opaque material only (or mainly) from one of the transverse sinuses following bilateral carotid injections. Total $10 \mathrm{ml}$ of non-ionic contrast agent (Optiray 300, Mallinckrodt Saglik, Istanbul, Turkey) was injected from each internal carotid artery at a rate of $5 \mathrm{ml} /$ second by automatic power injector (Liebel-Flarsheim, Angiomat Illumena, Cincinnati, USA). The imaging process, as well as arterial and capillary phase, was followed up from starting venous phase to filling the vena jugularis interna. We examined the venous sinus's filling time and diameter, and whether they had been filled by all injections. The venous drainage dominance was then divided into three groups: right dominance, left dominance and co-dominance (Figures 1, 2).

Firstly, we assessed whether there were any statistically significant associations between venous drainage dominance

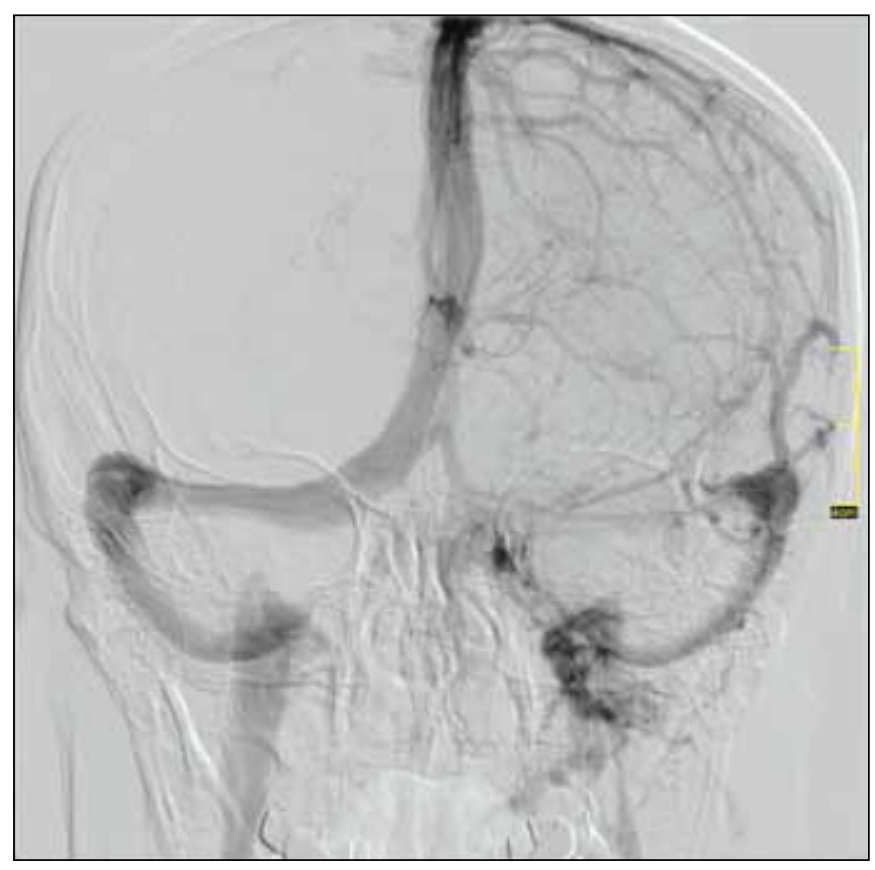

Figure 1: Left ICA injections and right venous dominance.

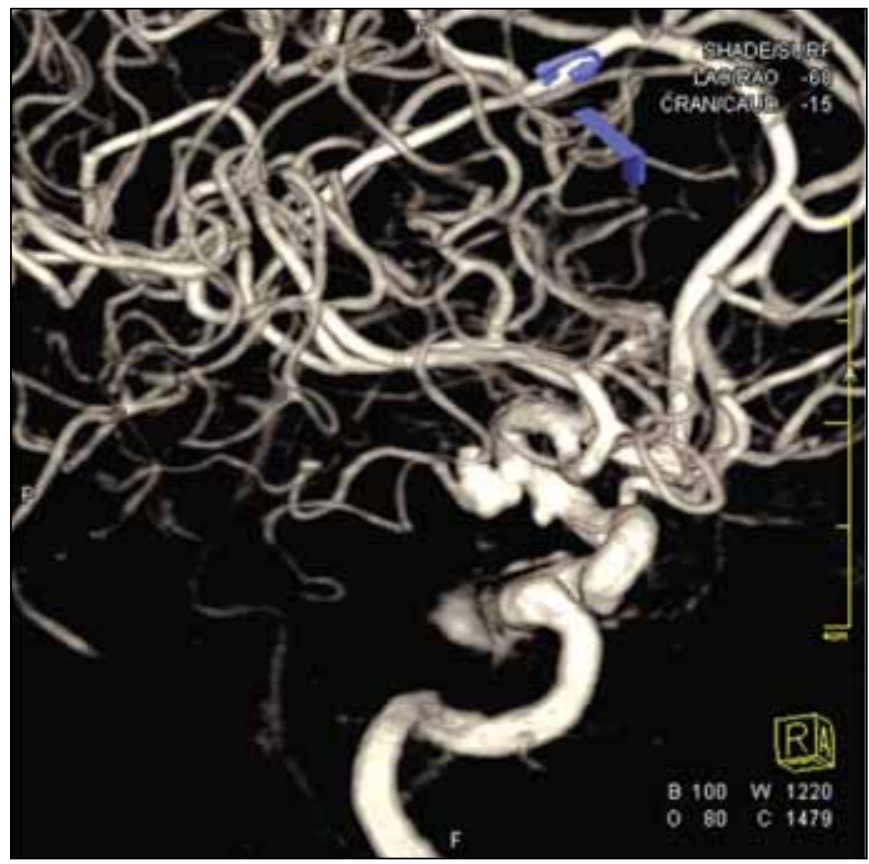

Figure 2: Patient, who had right venous dominance, had aneurysms at posterior communicating artery and ICA that were demonstrated with the right ICA injections. 
and aneurysm formation and ruptured aneurysms. We also assessed statistically significant associations between aneurysm formation and other parameters such as age, sex, smoking, and hypertension.

All analyses were done using the SPSS 9.0 (SPSS for Windows 9.0, Chicago, IL) software package. Continuous variables were presented as mean \pm standard deviation (SD), whereas categorical variables were presented as frequencies. The aneurysm's location, size, D/N, dominance of venous drainage, aneurysm sides, smoking, hypertension, Fisher scores and WFNS scores of associations between categorical variables were tested with the Pearson $\mathrm{X}^{2}$ (chi-squared test) test. In order to test whether the data were normally distributed, the Kolmogorov-Smirnov test was applied. Correlations between the parameters and the association with other variables were studied using Pearson's correlation coefficient if the data met the criteria for normal distribution, or otherwise using the Spearman correlation test. A p value less than 0.05 was considered as significant.

\section{- RESULTS}

Cerebral DSA was performed in 86 patients and the total number of aneurysms was 105 . We found that 34 patients $(39.50 \%)$ were male, 52 patients $(60.50 \%)$ were female and the average age was $56.22 \pm 11.10$ years. Seventyfive aneurysms were ruptured and 30 aneurysms were unruptured. Thirty aneurysms (28.6\%) were located at the bifurcation of the middle cerebral artery, 22 aneurysms (21\%) were internal carotid artery (ICA) (two aneurysms were on the cervical segment, six aneurysms were on the para-ophthalmic segment, six aneurysms were on the cavernous segment, eight aneurysms were on the supra-clinoid segment), 37 aneurysms (35.2\%) were on the anterior communicating artery, nine aneurysms (8.6\%) were on the posterior communicating artery, one aneurysm was (1.0\%) posterior cerebral artery and four aneurysms $(3.8 \%)$ were basilar artery, two aneurysms $(1.9 \%)$ were on the posterior inferior cerebellar artery. Fortysix aneurysms were demonstrated from right carotid artery injections and 52 aneurysms were demonstrated from the left carotid artery injections; the others (7 aneurysms) were demonstrated from the vertebral artery injections. Thirty-nine patients had right dominant brain venous drainage, 19 patients had left dominant brain venous drainage and 28 patients had co-dominant brain venous drainage. The aneurysm's size was minimum $1.2 \mathrm{~mm}$ and the maximum was $30 \mathrm{~mm}$ (average:
$7.07 \pm 4.8 \mathrm{~mm})$. The aneurysms' $\mathrm{D} / \mathrm{N}$ values ranged from a minimum of 0.6 to a maximum of 4 (average: $2.01 \pm 0.70$ ). Fisher and WFNS scores were recorded in 67 patients; 3 patients (3.5\%) had Fisher grade I, 23 patients (26.7\%) had Fisher grade II, 19 patients (22.10\%) had Fisher grade III and 22 patients $(25.60 \%)$ had Fisher grade IV hemorrhage. Eighteen (20.90\%) patients had WFNS score I, 23 (26.7\%) patients had WFNS score II, 6 (7\%) patients had WFNS score III, 19 (22.10\%) patients had WFNS score IV, one (1.2\%) patient had WFNS score V. Fifty patients had hypertension (58.10\%) and 70 patients were smoking (81.40\%).

The most common aneurysm localization was the anterior communicating artery, followed by the middle cerebral artery bifurcation. However, localization is not statistically significant for rupture in our study. There is no statistically significant association between the aneurysm's size and rupture. There is also no association between age and aneurysm formation or rupture. There is a negative correlation between $\mathrm{D} / \mathrm{N}$ ratio and Fisher $(r=-0.273, p=0.018)$ scores. Fisher scores show a positive correlation with WFNS scores $(r: 0.617, p<0.001)$. There is a statistically significant association between venous dominance and aneurysm $(p<0.001)$. If venous dominance is on the right side, aneurysms are also usually demonstrated from the right carotid artery injections; or if venous dominance is left side, aneurysms are also usually demonstrated from the left carotid artery injections (Table I). We also evaluated venous dominance in ruptured aneurysms. There is a statistically significant association between venous dominance and rupture $(p<0.001)$ (Table II). It means that patients, who had ruptured aneurysms that were demonstrated from right or left ICA injections, also had ipsilateral venous dominance. If ruptured aneurysms are demonstrated from right ICA injections, venous dominance is usually the right side or if ruptured aneurysms are demonstrated from left ICA injections venous dominance is usually the left side.

Demographic properties of the patients can be seen in Table III.

There is also a positive correlation between hypertension and rupture $(r=0.236, p=0.015)$.

\section{DISCUSSION}

Risk factors for SAH due to aneurysm are hypertension, smoking, using cocaine, using oral contraceptives, pregnancy, excessive alcohol use, diurnal changes in blood pressure,

Table I: Association Between Venous Dominancy and Sides of Aneurysm

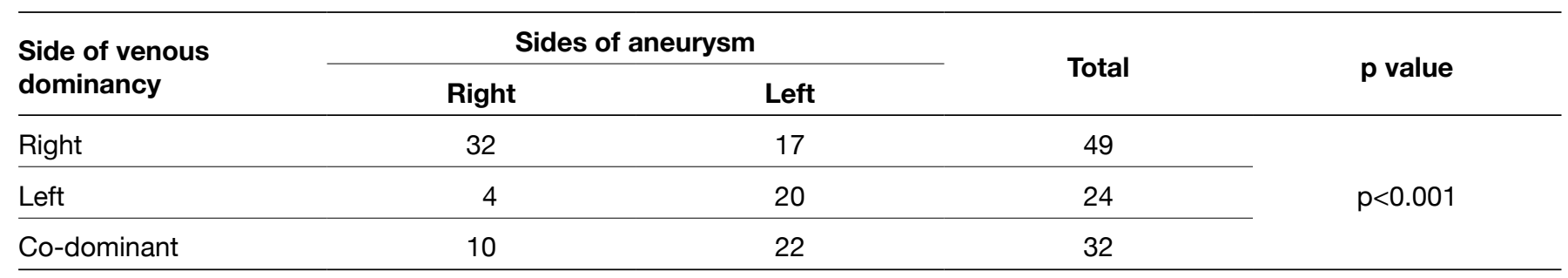

Chi-Square Test was used. 
Table II: Association Between Venous Dominancy and Sides of Aneurysm in Patients Who had Ruptured Aneurysm

\begin{tabular}{lcccc}
\hline \multirow{2}{*}{$\begin{array}{l}\text { Side of venous } \\
\text { dominancy }\end{array}$} & \multicolumn{2}{c}{ Sides of aneurysm } & Total & p value \\
\cline { 2 - 4 } & Right & 19 & 13 & 32 \\
\hline Right & 0 & 17 & 17 & $\mathrm{p}<0.001$ \\
\hline Left & 8 & 18 & 26 & \\
\hline Co-dominant & 19 & Left & \\
\hline
\end{tabular}

Chi-Square Test was used.

Table III: Demographic Properties of the Patients

\begin{tabular}{|c|c|c|}
\hline \multicolumn{2}{|l|}{ Patient number } & 86 \\
\hline \multicolumn{3}{|l|}{ Sex } \\
\hline \multicolumn{2}{|l|}{ Female } & $52(60.5 \%)$ \\
\hline \multicolumn{2}{|l|}{ Male } & $34(39.5 \%)$ \\
\hline \multicolumn{2}{|l|}{ 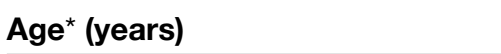 } & $56.22 \pm 11.10$ \\
\hline \multirow[t]{2}{*}{ Smoking } & + & $70(81.40 \%)$ \\
\hline & - & $16(18.60 \%)$ \\
\hline \multirow[t]{2}{*}{ Hypertension } & + & $50(58.1 \%)$ \\
\hline & - & $36(41.9 \%)$ \\
\hline \multicolumn{2}{|l|}{ Aneurysm number } & 105 \\
\hline \multirow[t]{2}{*}{ Aneurysm rupture } & + & 75 (71.4\%) \\
\hline & - & $30(28.6 \%)$ \\
\hline \multicolumn{3}{|l|}{ Venous dominancy } \\
\hline \multicolumn{2}{|l|}{ Right } & $36(45.3 \%)$ \\
\hline \multicolumn{2}{|l|}{ Left } & $19(22.1 \%)$ \\
\hline \multicolumn{2}{|l|}{ Co-dominancy } & $28(32.6 \%)$ \\
\hline \multicolumn{2}{|l|}{ Aneursym's size ${ }^{\star}$} & Average: $7.07 \pm 4.8 \mathrm{~mm}$ \\
\hline \multicolumn{2}{|c|}{ Dome and neck ratio $(\mathrm{D} / \mathrm{N})$ * } & Average: $2.01 \pm 0.70$ \\
\hline \multicolumn{3}{|c|}{ Aneurysm location } \\
\hline \multicolumn{2}{|l|}{ bifurcation } & $30(28.6 \%)$ \\
\hline \multicolumn{2}{|c|}{ internal carotid artery } & $22(21 \%)$ \\
\hline \multicolumn{2}{|c|}{ anterior communicating artery } & $37(35.2 \%)$ \\
\hline \multicolumn{2}{|c|}{$\begin{array}{l}\text { posterior communicating } \\
\text { artery }\end{array}$} & $9(8.6 \%)$ \\
\hline \multicolumn{2}{|c|}{ posterior cerebral artery } & $1(1.0 \%)$ \\
\hline \multicolumn{2}{|c|}{ basilar artery } & $4(3.8 \%)$ \\
\hline \multicolumn{2}{|c|}{$\begin{array}{l}\text { posterior inferior cerebellar } \\
\text { artery }\end{array}$} & $2(1.9 \%)$ \\
\hline
\end{tabular}

*: Values are presented as mean $\pm S D$.

family history of $\mathrm{SAH}$ and aneurysm, genetic disorders, older ages, lumbar puncture and cerebral angiography in patients with aneurysm (14). The life-threatening and debilitating consequences of SAH may be prevented if the aneurysm can be occluded before rupture. For effective management of patients with un-ruptured aneurysms, prognostic criteria for the risk of rupture are needed of which aneurysm size, location, and multiplicity are key prognostic factors.

Data from a retrospective International Study of Un-ruptured Intracranial Aneurysms (ISUIA) calculated a much lower risk of rupture (0.05/year) of aneurysms less than $10 \mathrm{~mm}$ in diameter when the patient is asymptomatic (14). Data from a prospective ISUIA study showed a risk of rupture of $0.52 \%$ for aneurysms $7-12 \mathrm{~mm}$ in diameter of the anterior circulation and $2.9 \%$ for aneurysms located in the posterior circulation in the same size category (15).

Although the prevention of hemorrhage has been advocated in recent times as the most effective strategy aimed at lowering mortality rates, management decisions require an accurate assessment of the risk of various treatment options compared with the natural history of the un-ruptured intracranial aneurysms (UIA) $(2,10)$. The natural history of the UIA and treatment outcomes are influenced by factors such as previous aneurysmal $\mathrm{SAH}$, age of patients, aneurysm characters such as size, location, and morphology, and experience of the medical treatment team.

Orz and Al Yamany (11) reported that the majority of aneurysms had ruptured while they were small in size and there was no size threshold beyond which the incidence of rupture of aneurysm increased. The majority of the small ruptured intracranial aneurysms located at the anterior communicating artery and distal anterior cerebral artery that should be considered as dangerous locations for aneurysm rupture, due to hemodynamic stress, and un-ruptured aneurysms of any size at these locations should be considered for treatment. Although the aneurysm size is a key factor in determining the risk of rupture, other factors such as aneurysm location and multiplicity should be considered for treatment strategies of un-ruptured intracranial aneurysms. They argued that they cannot consider that small aneurysms are safe lesions and they should be considered for treatment, especially those located at areas of high hemodynamic stress (11). In our study, the most common aneurysm location was the anterior communicating artery (35.2\%) and the middle cerebral artery was the second (28.6\%). However, localization was not found to be statistically significant for rupture in our study.

Kassel and Torner (6) found that $13 \%$ of ruptured aneurysms were less than $5 \mathrm{~mm}$, while $57 \%$ were between 5 and $10 \mathrm{~mm}$ in diameter. They concluded that the un-ruptured aneurysms less than $10 \mathrm{~mm}$ in diameter could not be considered innocuous and operation should be considered for lesions more than 
$5 \mathrm{~mm}$ in diameter (6). In our study, the aneurysm's size was minimum $1.2 \mathrm{~mm}$ and maximum $30 \mathrm{~mm}$ (average: $7.07 \pm 4.8$ $\mathrm{mm}$ ) and contrary to studies in the literature, there was no statistically significant association between the aneurysm's size and incidence of rupture.

As a means of providing an inclusive risk prediction model, the PHASES score, based on a pooled analysis of six prospective natural history studies, was recently proposed. Overall, the authors incorporated 8,382 patients with 10,272 un-ruptured intracranial aneurysms. Mean patient age was 60 years, and $68 \%$ of the patients were women. The overall annual rupture rate was $1.4 \%$ (95\% confidence interval, $1.1 \%$ to $1.6 \%$ ). After a multivariable Cox regression analysis, the authors derived a rupture prediction model based on population, hypertension, age, size of the aneurysm, earlier subarachnoid hemorrhage from another aneurysm, and site of the aneurysm - PHASES (4).

Age, aneurysm size and sex were not associated with rupture in our study but there was a positive correlation between hypertension and rupture, as expressed in the literature.

We did not assess the aneurysm's shape for any association with risk of rupture. We only evaluated the aneurysm's size and $\mathrm{D} / \mathrm{N}$ ratio regarding the aneurysm's morphology. There are many studies in the literature about the association of the aneurysm's morphology and rupture. The shape of the aneurysmal sac has a close relationship with rupture, like the size of the aneurysm. The fundus is generally spherical and smooth. If an aneurysm is lobular, with loculus, flow direction, with a wide neck and dome, it bleeds easily. A short and wide aneurysm sac tears more easily than a long and narrow one. A rough-wall aneurysm (31\%) has almost twice the risk of bleeding than an aneurysm with a smooth wall (14\%) (12). There was a negative correlation between the $\mathrm{D} / \mathrm{N}$ ratio and Fisher scores in our study. It means that if an aneurysm's dome decreased or the neck increased, the amount of bleeding would increase, and vice versa. When we see a wide-necked aneurysm we should surely treat it because of the increased bleeding risk.

In our study, there was a statistically significant association between venous dominance and the aneurysmal side. If venous dominance was in the right side, aneurysms were also usually demonstrated with right carotid artery injections; or if venous dominance was in the left side, aneurysms were also usually demonstrated with left carotid artery injections. This may mean that venous dominance can also be a predisposing factor for aneurysm formation, similar to hypertension, smoking, genetic disorders and other parameters. Venous dominance also had a statistically significant association with rupture in our study. If patients had right-left venous dominance and an aneurysm that was demonstrated from ipsilateral injections, they tended to be more likely to rupture than the other side aneurysms. Perhaps venous dominance can also be a predictive sign for rupture. Brain venous dominance is a subject that many investigators have studied so far but studies were limited in that they were generally performed on cadavers. Venous drainage may be evaluated with computed tomography but we used DSA because we can assess the all phases of venous drainage in this way. The right venous dominance rate was $45 \%$, left venous dominance rate $22 \%$ and co-dominance rate is $33 \%$ in this study and these results are not different from the literature. There are some studies in the literature about venous drainage, but most of them are about the anatomy and possible venous anomalies. $\mathrm{Ma}$ et al. assessed the association between the surgical risk of cavernous malformation and venous anomalies (9). Chi et al. evaluated chronic influences such as obstructive sleep apnea on cerebral venous flow (3). There is no study in the literature examining the link between brain venous drainage and aneurysm formation or rupture.

There are some limitations of this study such as the small number of patients and inadequate physiological information on the association of venous dominance and aneurysm formation- rupture.

\section{- CONCLUSION}

Venous dominance may be a predisposition for aneurysm or may be a predictive factor for rupture. Cerebral aneurysm formation and rupture are multifactorial and subject to various predisposing factors such as hypertension, smoking, using cocaine, using oral contraceptives, pregnancy, excessive alcohol use, diurnal changes in blood pressure, family history of SAH and aneurysm, genetic disorders, and older age. Our patients may simultaneously have more than one of these factors. This is a unique study examining the link between brain venous drainage and aneurysm formation or rupture. This association might be assessed in larger series as a distinct entity in vivo or in vitro.

\section{- REFERENCES}

1. Boswell S, Thorell W, Gogela S, Lyden E, Surdell D: Angiogram-negative subarachnoid hemorrhage: Outcomes data and review of the literature. J Stroke Cerebrovasc Dis 22: 750-757, 2013

2. Broderick JP, Brott TG: Initial and recurrent bleeding is the leading cause of death following subarachnoid hemorrhage. Stroke 25: 1342-1347, 1994

3. Chi HY, Lin CS, Hsu MH, Chan PC, Hu HH: Chronic influences of obstructive sleep apnea on cerebral venous flow. J Ultrasound Med 34: 2043-2048, 2015

4. Greving JP, Wermer MJ, Brown RD Jr, Morita A, Juvela S, Yonekura M, Ishibashi T, Torner JC, Nakayama T, Rinkel GJ, Algra A: Development of the PHASES score for prediction of risk of rupture of intracranial aneurysms: A pooled analysis of six prospective cohort studies. Lancet Neurol 13: 59-66, 2014

5. Jung JY, Kim YB, Lee JW, Huh SK, Lee KC: Spontaneous subarachnoid haemorrhage with negative initial angiography: A review of 143 cases. J Clin Neurosci 13: 1011-1017, 2006

6. Kassell NF, Torner JC: Size of intracranial aneurysms. Neurosurgery 12: 291-297, 1983

7. Kissela BM, Saurbeck L, Wood D, Khoury J, Carrozzella J, Pancioli A, Jauch E, Moomaw CJ, Shukla R, Gebel J, Fontaine $\mathrm{R}$, Broderick J: Subaracnoid hemorrhage: A preventable disease with a heritable component. Stroke 33:1321-1326, 2002 
8. Kocaeli H, Korfalı E: Subaraknoid kanama: Anevrizmalar. Norobilimde Bugun 1:1-6, 2003 (In Turkish)

9. Ma L, Chen XL, Ma J, Zhao YL: The associated venous anomalies variant and adjacent brain function on iron sensitive image indicate surgical risk of cavernous malformation. Neurol Med Chir 56: 9-20, 2016

10. Orz YI, Hongo K, Tanaka Y, Nagashima H, Osawa M, Kyoshima K, Kobayashi S: Risks of surgery for patients with unruptured intracranial aneurysms. Surg Neurol 53: 1-29, 2000

11. Orz Y, Al Yamany M: The impact of size and location on rupture of intracranial aneurysms. Asian J Neurosurg 10: 26-31, 2015

12. Suvela S, Porrasm, Poussa K: Natural history of unruptured intracranial aneurysm probability of and risk factors for aneurysm rupture. J Neurosurg 96: 57-65, 2002
13. Uddin MA, Haq TU, Rafique MZ: Cerebral venous system anatomy. J Pak Med Assoc 56: 516-519, 2006

14. Unruptured intracranial aneurysms-risk of rupture and risks of surgical intervention. International study of unruptured intracranial aneurysms investigators. $\mathrm{N}$ Engl $\mathrm{J}$ Med 339: 1725-1733, 1998

15. Wiebers DO, Whisnant JP, Huston J, Meissner I, Brown RD, Piepgras DG, Forbes GS, Thielen K, Nichols D, O'Fallon WM, Peacock J, Jaeger L, Kassell NF, Beckman GL, Torner JC: Unruptured intracranial aneurysms: Natural history, clinical outcome, and risks of surgical and endovascular treatment. Lancet 362: 103-110, 2003 